\title{
DỨBin
}

Technological University Dublin

ARROW@TU Dublin

2009-06-10

\section{Performance Evaluation of Antennas for UWB Radar and Positioning Systems}

\author{
Antoine Dumoulin \\ Technological University Dublin, antoine.dumoulin@gmail.com \\ Matthias John \\ Technological University Dublin, matthias.john@tudublin.ie \\ Patrick McEvoy \\ Technological University Dublin, patrick.mcevoy@tudublin.ie
}

See next page for additional authors

Follow this and additional works at: https://arrow.tudublin.ie/ahfrccon

\section{Recommended Citation}

A. Dumoulin, et al. (2009) Performance Evaluation of Antennas for UWB Radar and Positioning Systems. Irish Signals \& Systems Conference, Dublin, Ireland, 10/06/2009, doi:10.1049/cp.2009.1729

This Conference Paper is brought to you for free and open access by the Antenna \& High Frequency Research Centre at ARROW@TU Dublin. It has been accepted for inclusion in Conference Papers by an authorized administrator of ARROW@TU Dublin. For more information, please contact arrow.admin@tudublin.ie, aisling.coyne@tudublin.ie,gerard.connolly@tudublin.ie.

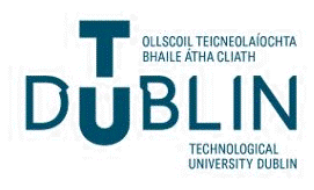


Authors

Antoine Dumoulin, Matthias John, Patrick McEvoy, and Max Ammann

This conference paper is available at ARROW@TU Dublin: https://arrow.tudublin.ie/ahfrccon/4 


\title{
Performance Evaluation of Antennas for UWB Radar and Positioning Systems
}

\author{
Antoine Dumoulin, Matthias John*, \\ Max J. Ammann* and Patrick McEvoy
}

\author{
*Centre for Telecommunications Value-Chain Research (CTVR) \\ School of Electronic and Communications Engineering,
}

Dublin Institute of Technology, Kevin St., Dublin 8, Ireland

email:antoine.dumoulin@student.dit.ie,max.ammann@dit.ie

\begin{abstract}
This paper presents a study of the performance of three antennas for ultra wideband radio ranging systems. By using different pulse types, the performance of the antennas is measured and quantified by using the Fidelity Factor.
\end{abstract}

Keywords - Pulse communication, UWB antenna, Fidelity Factor.

\section{INTRODUCTION}

Ultra Wideband (UWB) radio technology is employed in communication, imaging, and radar systems. The main advantages of UWB radio stems from the wide bandwidth up to $7.5 \mathrm{GHz}$ and from the low power levels. This wide bandwidth can reduce susceptibility to multipath fading, increase system capacity, and improve range resolution for precision positioning measurement. The reduced power spectral density offers a low probability of detection and low interference to other services, enabling smaller re-use distances. UWB radio is regulated and reviewed by several organizations such as the Federal Communications Commission (FCC), the European Conference of Postal and Telecommunications Administration (CEPT), or the Institute of Electrical and Electronic Engineers (IEEE). In 2002 the FCC, allowed the use of UWB devices in North America [1]. This commission has established that the frequency spectrum of the UWB radio has to be between $3.1 \mathrm{GHz}$ and $10.6 \mathrm{GHz}$ for handheld UWB devices with an Equivalent Isotropically Radiated Power (EIRP) of -41.3 $\mathrm{dBm} / \mathrm{MHz}$. The FCC specifies, at any point in time, the fractional bandwidth has to be equal to or greater than $20 \%$ or to have a $500 \mathrm{MHz}$ bandwidth regardless of the fractional bandwidth. This ultra wide bandwidth provides great opportunity for very high transfer data rate as well as precision ranging with very low power levels, which are considered noise by other communication systems. Hence it is possible to use this technology without interfering with other systems such as GPS, Bluetooth and wireless radio standards for Wireless Local Area Network (802.11a, 802.11b, 802.11g, and the $802.11 n)$.

For the European Union, CEPT specify that until December 2010, UWB devices without Detect And Avoid system (DAA) and Low Duty Cycle (LDC) can work in the 4.1-4.8 GHz and 6-8.5 GHz (no date restriction) frequency range [2]. If one of these systems are implemented then the 3.1-4.8 GHz and the $6-9 \mathrm{GHz}$ frequency range is accessible. Hence by its characteristic, UWB radio is suitable for 802.15.3a [3] (High Rate Wireless Personal Area Networks) and 802.15.4a [4] (low-rate Wireless Personal Area Networks) standards. The UWB radio signal can be generated by two dominant modulation schemes, by Direct Sequence Ultra-Wide Band DSUWB, or Orthogonal Frequency Domain Multiplexing (OFDM) [5]. For OFDM the spectrum mask can be divided in 5 channels each containing 3 sub-bands, each of $528 \mathrm{MHz}$, except for the last channel which contain 2 sub-bands. The 802.15.4a has been the first international standard to establish a wireless physical layer for precision ranging. 
Impulse radio (IR) UWB can be used for communication with an optional ranging capability, while the Chirp Spread Spectrum (CSS) is generally used for communications. The IR UWB radio employs a very short pulse to transmit the information, therefore it is essential that the pulse leaving the antenna remains as similar as possible to the input pulse. Moreover IR-UWB uses narrow pulses, which translate to a wide spectrum in the frequency domain. While these characteristics enable high performance from a communication, ranging, or imaging point of view, it creates challenges for an antenna designer point of view.

This paper presents a method to measure the time domain performance of an antenna. The method is applied to a rectangular planar monopole and to two optimised spline-shaped monopole antennas.

\section{S-PARAMETERS}

For pulse systems, the antenna has to be well matched over a bandwidth (BW) at least equal to that of the BW of the pulse. This antenna must have an input reflection coefficient (S11) below -10 dB over the whole bandwidth. Moreover the antenna has to be small and easily fabricated for low-cost. Hence the most appropriate candidates are planar monopoles and dipoles. They are usually built from standard copper clad PCB material and are fed by using microstrip or co-planar-waveguide printed transmission lines. Microstrip is one of the most popular types of transmission lines because it can be easily integrated with other microwave devices. While the S11 magnitude is important for good matching, the S21 phase linearity is more relevant for a pulse communication system. For a pulse communication system, it is important that the radiated pulse from the antenna suffers no distortion. Hence if the S21 phase is not linear over the full frequency range, the pulse fidelity will not be preserved in the antenna.

\section{ANTENNA GEOMETRIES AND OPTIMISATION}

The three antennas used in this study have been optimised using different conventional and evolutionary optimisation algorithms. The first antenna (A) is a simple rectangular monopole which was optimised for wide-band performance using classic parameter sweep and interpolation techniques.

The geometries of antennas $\mathrm{B}$ and $\mathrm{C}$ are based on a quadratic Bezier spline outline [6] and optimised by evolutionary optimisation methods. The spline is constructed from quadratic curve segments which are defined by a number of control points. By moving these points, the shape of the geometry and hence the performance of the antenna can be changed. Both the radiating element and the groundplane are constructed in this way.

Two different optimisation algorithms have been used to arrive at a final geometry for the antennas. Antenna B was optimised by a Genetic Algorithm [6] and antenna $\mathrm{C}$ by a more efficient Evolutionary Global Optimisation algorithm [7]. These algorithms take the coordinates of the control points as parameters and evolve them towards a performance goal. This optimisation goal consists of a combination of bandwidth (which has to be maximised) and lower edge frequency (i.e. the start of the band, which has to be minimised). The goal is computed by full wave electromagnetic simulation using the time domain solver of CST Microwave Studio [8]. Figure 1 shows the final geometries for the rectangular monopole (A), the GA (B) and Parallel EGO (C) optimised monopole antennas.

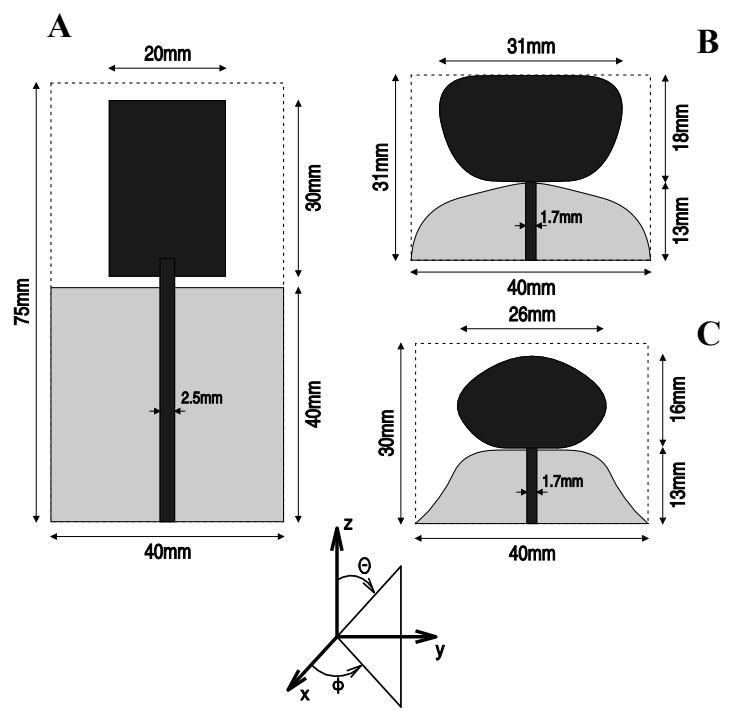

Figure 1: Geometries of the rectangular plate monopole (A), GA optimised monopole (B) and ParEGO optimised monopole $(\mathrm{C})$.

\section{ANTENNA TRANSFER FUNCTIONS}

Figure 2 and Figure 3 show the S11 phase/magnitude and the input and radiated signal from antennas A, B and C. A simulated $7.5 \mathrm{GHz}$ bandwidth Gaussian signal centred at $6.85 \mathrm{GHz}$ was applied at the input port and an electric-field virtual probe was placed at a distance of $300 \mathrm{~mm}$ from the antennas. It is clear that the antennas do not have linear S11 phase, but antennas $\mathrm{B}$ and $\mathrm{C}$ are better matched than antenna $\mathrm{A}$. 


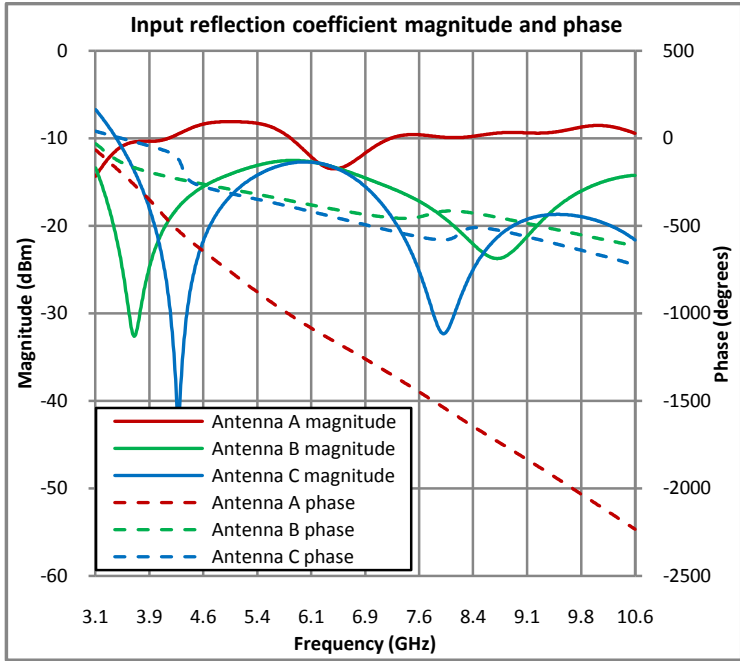

Figure 2: S11 phase and magnitude for Antennas A, $\mathrm{B}$ and $\mathrm{C}$.

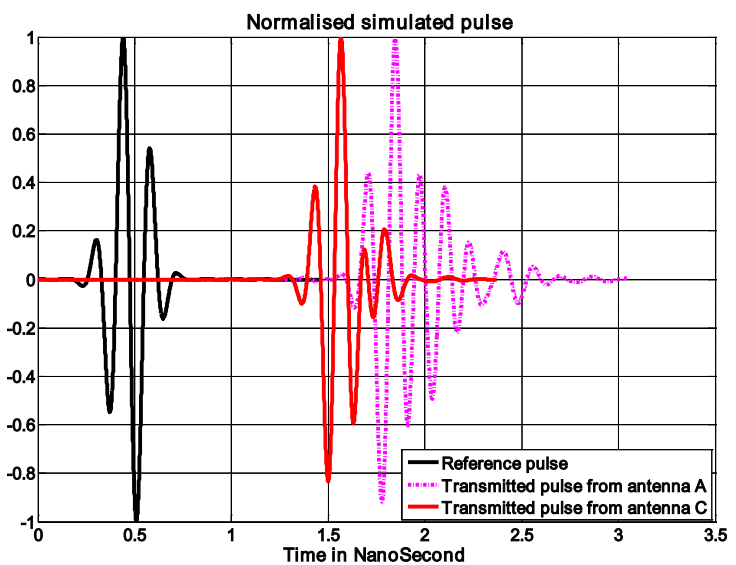

Figure 3 : Simulated reference and probe pulse signals.

While the S11 parameter is useful from a matching point of view, the magnitude and phase of the transfer function is more useful for an evaluation of the time domain performance. The transfer function $(T F(f))$ can be calculated as follow [9]:

$T F(f)=\frac{F r(t)}{F i(t)}$

Where $\operatorname{Fr}(t)$ is the Fourier Transform of the radiated signal and $F i(t)$ is the Fourier transform of the input signal.

These functions are interesting because they show how the radiated signal differs from the input reference signal in the frequency domain. Any variation in the transfer function will result in signal distortion. Hence it is essential that the magnitude and phase transfer functions are respectively, as flat and linear as possible. By looking at Figure 3, it is seen that the signal transmitted from antenna $\mathrm{C}$ is a closer replica to the reference than from antenna A. Figure 4 shows the transfer function magnitude rate of change while Figure 5 shows the transfer function phase rate of change. On both plots, it is obvious that the antenna $\mathrm{B}$ and $\mathrm{C}$ transfer function exhibits better characteristics than the antenna A transfer function, such as smaller magnitude and phase variation and improved linearity. These differences in the transfer function explain why the probe received signals from antenna $\mathrm{A}$ are more distorted than from antenna $\mathrm{C}$ (see Figure 3)

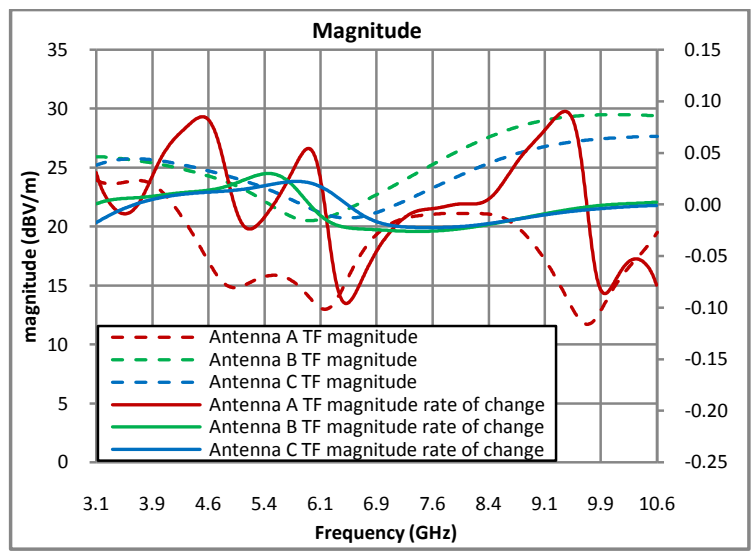

Figure 4: Magnitude transfer functions for Antennas A, $\mathrm{B}$ and $\mathrm{C}$.

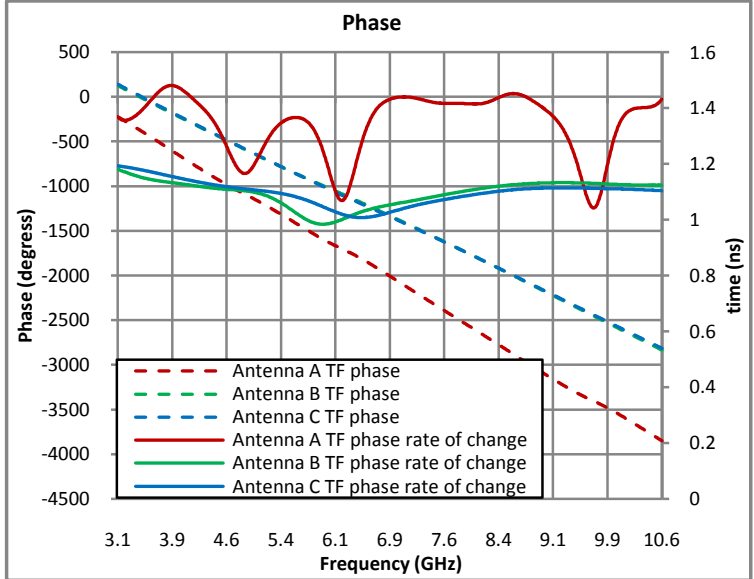

Figure 5: Phase transfer functions for Antennas A, $\mathrm{B}$ and $\mathrm{C}$.

\section{FIDELITY FACTOR}

Using the reference and received signals, it becomes possible to quantify the level of similarity between signals. The fidelity factor is used for this purpose and accounts for the antenna magnitude and phase transfer function. This factor is determined by the absolute value of the maximum of the cross correlation coefficient of the two normalised signals [10]. For a discrete waveform the fidelity factor can be defined as:

$$
F F=\max \left|\frac{\sum_{n=0}^{N-1} x_{1}(n) x_{2}(n+t)}{\sqrt{\sum_{n=0}^{N-1} x_{1}^{2}(n) \sum_{n=0}^{N-1} x_{2}^{2}(n)}}\right|
$$


While for an infinite waveform it is determined according to:

$$
F F=\max \left|\frac{\int_{-\infty}^{\infty} x_{1}(t) x_{2}(t+\tau) d t}{\sqrt{\int_{-\infty}^{\infty}\left|x_{1}(t)\right|^{2} d t} \sqrt{\int_{-\infty}^{\infty}\left|x_{2}(t)\right|^{2} d t}}\right|
$$

where $x_{1}$ symbolises the non-normalised input signal and $x_{2}$ the non-normalised radiated signal. The highest value is $1(100 \%)$, which means that both signals are identical. Because of the normalisation procedure the Fidelity Factor cannot provide any information about the signal amplitude. In Figure 6, the fidelity factor is plotted for the three antennas. These results have been made by placing virtual probes around the antenna azimuth plane. Both antennas have been excited by an amplitude modulated square root raised cosine pulse having a bandwidth of $7.5 \mathrm{GHz}(-10 \mathrm{~dB})$ and centred at 6.85 $\mathrm{GHz}$. This pulse has the characteristic of being optimal for the FCC UWB mask and has very low side lobes, which means that all the power is concentrated in the fundamental lobes [4]. The time domain function for a square root raised pulse is defined as follows:

$f(t)=\frac{2 \beta}{\pi \sqrt{\tau}} * \frac{\cos \left((1+\beta) \pi \frac{t}{\tau}\right)+\frac{\sin \left((1-\beta) \pi \frac{t}{\tau}\right)}{4 \beta \frac{t}{\tau}}}{1-\left(4 \beta \frac{t}{\tau}\right)^{2}}$

Where $\beta$ is the rolloff factor and $\tau$ is the pulse duration. Figure 7 shows the input signal and the radiated signal (65\% fidelity) from antenna $(\mathrm{A})$, in the time and frequency domain. It is clear that a radiated pulse having a $65 \%$ fidelity is significantly distorted. In fact, even a signal with $90 \%$ fidelity is quite distorted (see Figure 8).

By analyzing the plot on Figure 6, it is interesting to see that, for antenna (B), the Fidelity Factor is orthogonally symmetrical due to the antenna shape. Hence the FF provides good information about the antenna radiation fidelity in the space. For a "narrow" UWB signal, the antennas behave differently. For such signals the power is concentrated within $500 \mathrm{MHz}$ or so. Hence the pulse is less degraded by the antenna characteristics than for a full range wideband UWB pulse. The yields a better radiated pulse. In Figure 10 both antennas have been simulated by using a pulse captured from a prototype pulse generation board provided by Decawave Ltd. [11]. This pulse has been captured using an AGILENT Infinium DSO81204A oscilloscope. The signal, shown in Figure 9, is centred at $4 \mathrm{GHz}$ and has a $600 \mathrm{MHz}$ bandwidth. It can be seen that in this case the fidelity factor value is greater and less spatially dependent compared to those in Figure 6.

\section{ANTENNA SYSTEMS}

In a real communication environment with a link between two antennas the fidelity between the incident and received pulse must be as high as possible for IR-UWB. An antenna system consists of one transmitting antenna and at least one receiving antenna. In this test case the transmitting and receiving antenna are both identical and have been setup a distance of $300 \mathrm{~mm}$ apart. The transmitting antenna has been excited using the prototype pulse signal. The pulse is received by an identical antenna and captured using an AGILENT Infiniium DSO81204A oscilloscope. During the measurement the pair of antennas has been oriented in different positions and the fidelity factor has been computed for each orientation given in Table 1. It is assumed that the "back" of the antenna is the ground plane side and the "face" of the antenna is the monopole side. It is seen that the fidelity factor varies depending on orientation but it is clear that the best result is achieved when the antenna's ground planes are facing each other (Back to Back). Since the radiated pulse is a function of the antenna, and consequently its fidelity factor, the antenna system fidelity factor is approximately equal to the square of the probe's fidelity factor as described below:

$$
F F_{\text {Antenna system }} \cong F F_{\text {probe }}^{2}
$$

\section{CONCLUSION}

Several frequency-domain-optimised UWB planar antennas have been tested for time-domain performance for impulse radio UWB by using the Fidelity Factor as performance metric. The fidelity factor is improved when the antenna shape do not contain abrupt geometric features seen in polygons. Smooth spline shapes realise enhanced matched bandwidth and reduce pulse ringing due to discontinuities. It has also been demonstrated that the pulse fidelity is a function of the linearity of magnitude and phase of the transfer function, which are good indicators of fidelity performance. Moreover, fidelity is not only spatially dependent but also a function of the signal bandwidth.

\section{ACKNOWLEDGMENT}

The authors would like to acknowledge Decawave Ltd. for providing the prototype UWB pulse generation board. 


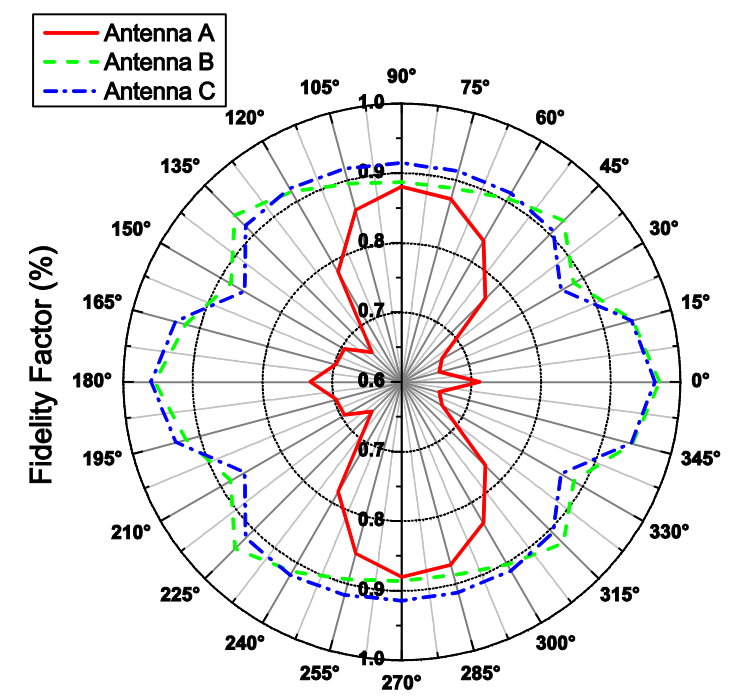

Figure 6: Fidelity factor as a function of azimuth angle for antennas $\mathrm{A}, \mathrm{B}$ and $\mathrm{C}$ excited with $7.5 \mathrm{GHz}$ bandwidth square root raised cosine pulse.
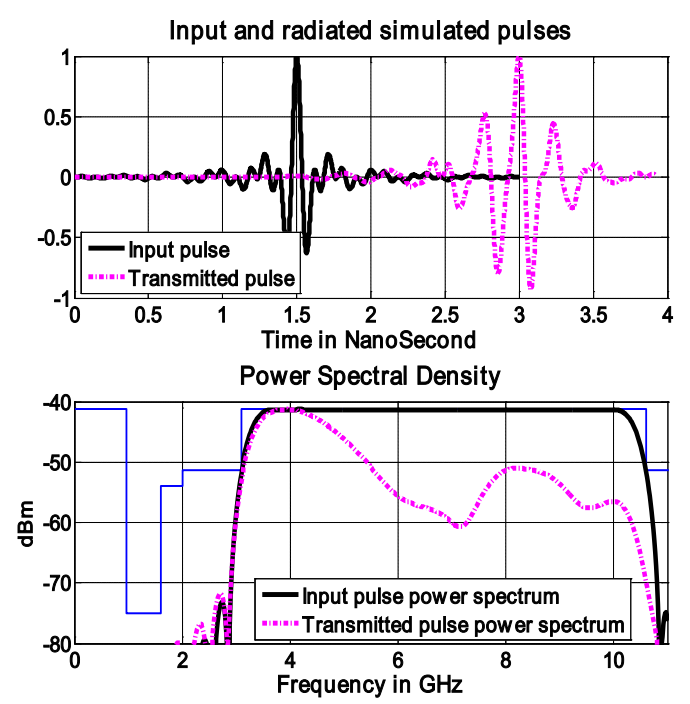

Figure 7: Time and frequency domain plot of a $65 \%$ fidelity transmitted $7.5 \mathrm{GHz}$ bandwidth square root raised cosine pulse.
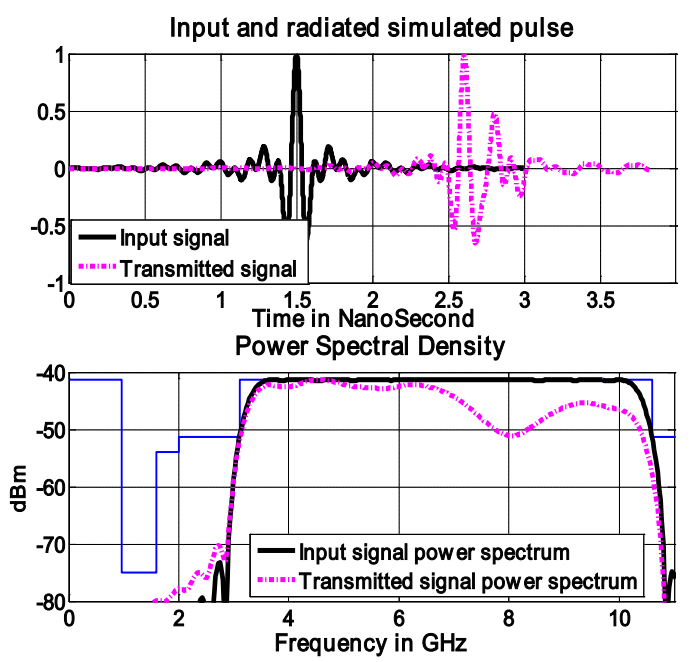

Figure 8: Time and frequency domain plot of a $90 \%$ fidelity transmitted $7.5 \mathrm{GHz}$ bandwidth square root raised cosine pulse.
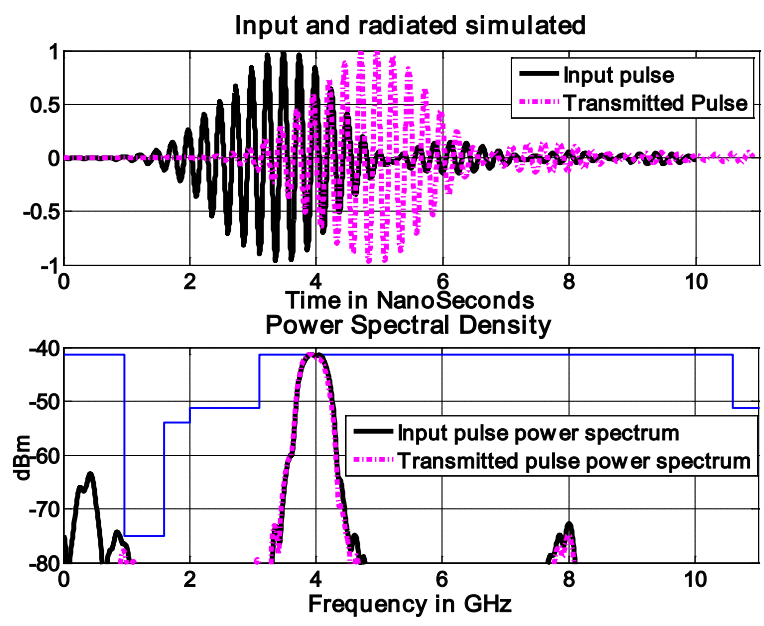

Figure 9: Time and frequency domain plot of $99.3 \%$ fidelity transmitted $600 \mathrm{MHz}$ Decawave pulse. 


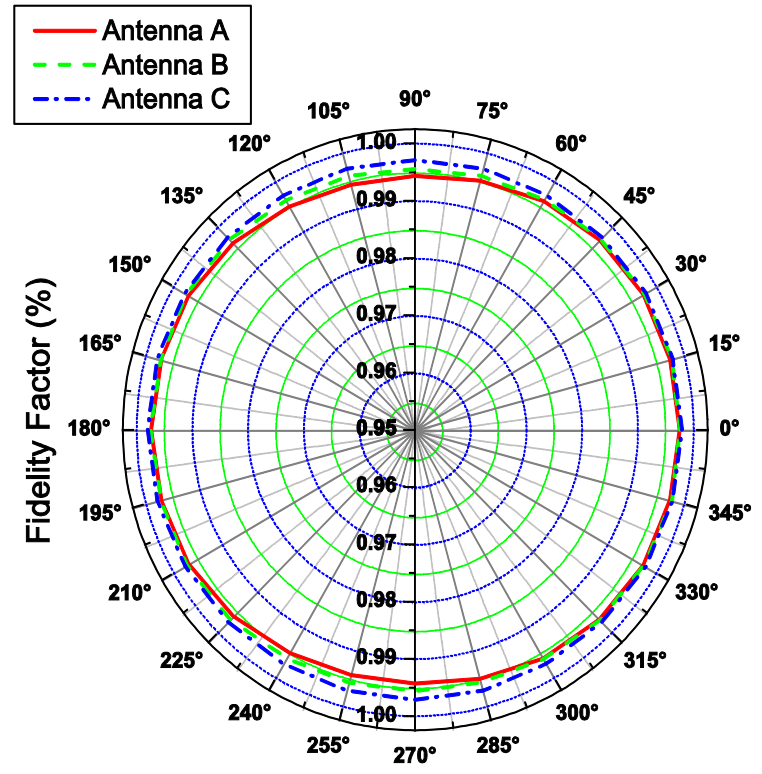

Figure 10: Fidelity factor as function of azimuth angle for Antennas A, B and C excited with the proprietary Decawave pulse.

\begin{tabular}{|l|l|l|l|l|l|l|}
\cline { 2 - 7 } \multicolumn{1}{c|}{} & $\begin{array}{l}\text { Back to } \\
\text { Back }\end{array}$ & $\begin{array}{l}\text { Back to } \\
\text { Side }\end{array}$ & $\begin{array}{l}\text { Back to } \\
\text { Face }\end{array}$ & $\begin{array}{l}\text { Face to } \\
\text { Face }\end{array}$ & $\begin{array}{l}\text { Face to } \\
\text { side }\end{array}$ & $\begin{array}{l}\text { Side by } \\
\text { Side }\end{array}$ \\
\hline Antenna A & 0.97367 & 0.95242 & 0.95958 & 0.94854 & 0.91734 & 0.92607 \\
\hline Antenna B & 0.99237 & 0.98480 & 0.98497 & 0.96894 & 0.98374 & 0.96407 \\
\hline Antenna C & 0.98249 & 0.96615 & 0.95684 & 0.95903 & 0.98897 & 0.97144 \\
\hline
\end{tabular}

Table 1: Measured Fidelity Factor for Antennas A, B and $\mathrm{C}$ excited with the proprietary Decawave pulse.

\section{REFERENCES}

[1] Federal Communication Commission, Part 15, First Report and Order, May 2002.

[2] The Commissions of the European Communities, "2007/131/EC Commission Decision of 21 February 2007 on allowing the use of the radio spectrum for equipment using ultra-wideband technology in a harmonised manner in the Community", Official Journal of the European Union, vol. 50, L 55, pp.33-36, February 2007

[3] IEEE Computer Society, 802.15.3 Part 15.3: Wireless Medium Access Control, (MAC) and Physical Layer (PHY), Specifications for High Rate Wireless and Personal Area Networks (WPANs), The Institute of Electrical and Electronics Engineers, 29 September 2003.

[4] IEEE Computer Society, Part 15.4: Wireless Medium Access Control (MAC) and Physical Layer (PHY) Specifications for Low-Rate Wireless Personal Area Networks
(WPANs), The Institute of Electrical and Electronics Engineers, 31 August 2007.

[5] K. Siwiak \& D. McKeown, (2004). Ultra Wideband Radio Technology. Chichester: John Wiley \& Sons Ltd.

[6] M. John and M.J. Ammann, "Spline Based Geometry for Printed Monopole Antennas," Electron. Lett., vol. 43 (6), pp. 317-319, 2007.

[7] M. John and M.J. Ammann, “Antenna Optimisation with a Computationally Efficient Multi-objective Evolutionary Algorithm," IEEE Trans. Antennas \& Propagation, AP 57, (1), 260-263, 2009.

[8] CST Microwave Studio, CST Computer Simulation Technology AG. [online] http://www.cst.com/

[9] N. Telzhensky and Y. Leviatan, "Novel Method of UWB Antenna Optimization for Specified Input Signal Forms by Means of Genetic Algorithm", IEEE Trans. Antennas \& Propagation, AP 54, (8), pp. 2216-2225, August 2006.

[10] D. Lamensdorf and L. Susman, "Basebandpulse-antenna techniques," IEEE Antennas Propag. Mag., vol. 36, no. 1, pp. 20-30, Feb. 1994.

[11] Decawave Ltd. [online] http://www.decawave.com/ 